\title{
BMJ Open Treatment of scabies using a tea tree oil- based gel formulation in Australian Aboriginal children: protocol for a randomised controlled trial
}

\begin{abstract}
Jackson Thomas, ${ }^{1}$ Rachel Davey, ${ }_{1}^{1}$ Gregory M Peterson, ${ }^{2}$ Christine Carson, ${ }^{3,4}$ Shelley F Walton, ${ }^{5}$ Tim Spelman, ${ }^{6}$ Tom Calma, ${ }^{7}$ Pascale Dettwiller, ${ }^{8}$ Jacinta Tobin, ${ }^{9}$ Faye McMillan, ${ }^{10}$ Paul Collis, ${ }^{1}$ Mark Naunton, ${ }^{1}$ Sam Kosari, ${ }^{1}$ Julia K Christenson, ${ }^{1}$ Andrew Bartholomaeus, ${ }^{1}$ John McEwen, ${ }^{1}$ Peter Fitzpatrick, ${ }^{11}$ Kavya E Baby ${ }^{12}$
\end{abstract}

To cite: Thomas J, Davey R, Peterson GM, et al. Treatment of scabies using a tea tree oil-based gel formulation in Australian Aboriginal children: protocol for a randomised controlled trial. BMJ Open 2018;8:e018507. doi:10.1136/ bmjopen-2017-018507

- Prepublication history and additional material for this paper are available online. To view these files, please visit the journal online (http://dx.doi. org/10.1136/bmjopen-2017018507).

Received 4 July 2017

Revised 31 October 2017

Accepted 8 November 2017

Check for updates

For numbered affiliations see end of article.

Correspondence to Dr Jackson Thomas; Jackson.Thomas@canberra. edu.au

\section{ABSTRACT}

Introduction In remote Aboriginal communities in Australia, scabies affects 7 out of 10 children before their first birthday. This is more than six times the rate seen in the rest of the developed world. Scabies infestation is frequently complicated by bacterial infection, leading to the development of skin sores and other more serious consequences, such as septicaemia and chronic heart and kidney diseases. Tea tree oil (TTO) has been used as an antimicrobial agent for several decades with proven clinical efficacy. Preclinical investigations have demonstrated superior scabicidal properties of TTO compared with widely used scabicidal agents, such as permethrin $5 \%$ cream and ivermectin. However, current data are insufficient to warrant a broad recommendation for its use for the management of scabies because previous studies were small or limited to in vitro observations.

Methods and analysis A pragmatic first trial will examine the clinical efficacy of a simple and low-cost TTO treatment against paediatric scabies and the prevention of associated secondary bacterial infections, with 1:1 randomisation of 200 participants (Aboriginal children, aged 5-16 years and living in remote Australia) into active control (permethrin 5\% cream) and treatment (5\% TT0 gel) groups. The primary outcome for the study is clinical cure (complete resolution). Secondary outcome measures will include relief of symptoms, recurrence rate, adverse effects, adherence to treatment regimen and patient acceptability.

Ethics and dissemination The project has received approvals from the University of Canberra Human Research Ethics Committee (HREC 16-133), WurliWurlinjang Health Service Indigenous subcommittee and the Aboriginal Medical Services Alliance Northern Territory reference group. The results of this study will be published in core scientific publications, with extensive knowledge exchange activities with non-academic audiences throughout the duration of the project. Trial registration ACTRN12617000902392; Pre-results.

\section{INTRODUCTION}

Scabies is a contagious, parasitic skin disease (dermatosis) caused by the acarine itch mite

\section{Strengths and limitations of this study}

- The study will be conducted in collaboration with a community-controlled Aboriginal Medical Service, in a clinically and culturally sound way with governance and approvals by expert Aboriginal and Torres Strait Islander researchers and organisations.

- A range of outcomes will be monitored, including clinical response, relief of symptoms, recurrence, adverse effects, adherence to treatment and patient acceptability.

- The educational and community support packages delivered to participating communities as part of this study will facilitate the effective control of scabies and offer long-term benefits to the community.

- This is a single-site study.

- Adherence to the treatment protocol may be suboptimal in an Aboriginal community setting in remote Australia; however, pragmatic strategies have been incorporated in the study design to promote adherence and limit attrition.

Sarcoptes scabiei var. hominis. Worldwide, there are 300 million cases of scabies each year, and the prevalence is as high as $>60 \%$ in Aboriginal children and about 25\% in Aboriginal adults in Australia. This is more than six times the rate seen in resource-poor communities worldwide but comparable with reported prevalence rates in Fiji. ${ }^{1-4}$ Scabies infestation has a negative impact on the quality of life of infected individuals, resulting in substantial stigmatisation and ostracism. ${ }^{56}$

S. scabiei releases antigens into the outer skin layer, resulting in local inflammatory and immune reactions, leading to severe itching (pruritus) and skin abrasions. ${ }^{78}$ Breaks in the epidermis serve as an entry point for bacteria, such as streptococci or staphylococci, and complement inhibitors released by scabies mites promote bacterial growth. 
This, in turn, can give rise to serious secondary infections. ${ }^{2}$ Superinfected lesions may develop into cellulitis or impetigo and may contribute to abscess formation. The sequelae predispose the individual to sepsis, and other serious consequences, such as chronic heart and kidney diseases. ${ }^{3}$ Australia's Northern Territory has the highest documented incidence of rheumatic heart disease in the world. Both chronic heart and kidney diseases are significant causes of premature mortality among Aboriginal Australians, contributing to a considerable life expectancy gap (10-17 years) between Aboriginal and non-Aboriginal people. $^{7}$

Most current scabicides are potentially hazardous and are associated with moderate to serious cutaneous and systemic side effects. ${ }^{3}{ }^{9-13}$ The safety of ivermectin (the sole oral therapy against scabies) has not been adequately established in the elderly, in patients with impaired liver function, in children aged $<5$ years or in pregnant women. No currently available acaricides possess ovicidal, antibacterial, anti-inflammatory and/or antipruritic properties. They are all ineffective at preventing treatment relapse arising from newly hatched mites, inflammatory skin reactions due to mite antigens and pyodermal progression.

Emerging resistance to existing scabies treatments raises concerns regarding their continuing effectiveness. ${ }^{14-17}$ In vitro sensitivity data for scabies mites over the last 10 years (Australian data) indicate that median survival times after treatment with the leading acaricides, ivermectin and permethrin, have increased twofold to threefold. Furthermore, treatment failures resulting from drug resistance have been documented. ${ }^{1415}$

In developed countries, scabies infestation affects individuals with both low and high income and social status. The disease can also represent an expensive burden for communities or institutions experiencing outbreaks (such as long-term care facilities, nursing homes, hospitals, schools and prisons). ${ }^{1819}$ A low-cost, topical treatment would be extremely valuable, especially in developing countries with low incomes and overcrowding, where tropical and subtropical climates may promote the occurrence of scabies infestation. ${ }^{1819}$

These public health concerns clearly demonstrate the need for further clinical studies into new antiscabietic agents. Tea tree oil (TTO) has shown promising results as an acaricide in preliminary in vitro studies. ${ }^{14}$ The therapeutic benefits of TTO-containing formulations for a range of dermatological conditions have been investigated in several small clinical studies, with mixed results. ${ }^{20} 21$ There have been concerns regarding the sensitisation potential of neat TTO; however, when the oil is formulated in a suitable pharmaceutical base (cream/ ointment/gel) containing concentrations $\leq 25 \%$, the risk of adverse skin reactions appears low. ${ }^{22}$ The potential of TTO toxicity in children is yet to be evaluated extensively. A recent randomised clinical trial found that TTO $(75 \% \mathrm{v} / \mathrm{v})$ was well tolerated in the 30-day treatment of the viral infection molluscum contagiosum in children (mean age $6.3 \pm 5.1$ years).$^{23}$ Irritation and sensitising potential of
TTO result largely from oxidised oil, due to elevated levels of peroxides and other TTO degradation products. ${ }^{24}$ In typical in-use conditions (amber glass bottle fitted with child-resistant polypropylene caps away from heat source and light), unformulated, neat TTO will have no appreciable degradation for up to 12 months. ${ }^{22} 24$ Due to its high volatility, $90 \%$ of the TTO evaporates quickly from the skin surface, minimising the potential for TTO components to travel into the deeper layers of the skin and to be absorbed into the bloodstream. However, terpinen-4-ol, $\alpha$-terpineol and 1,8-cineole can penetrate the epidermal layer of the skin sufficiently to produce antimicrobial, anti-inflammatory and potentially acaricidal effects. ${ }^{20} 25$

TTO has been shown to possess insecticidal, acaricidal and repellent properties against a range of medical and veterinary pests, such as house dust mites, ${ }^{26}$ Demodex mites, ${ }^{27}{ }^{28}$ swine mites ${ }^{29}{ }^{30}$ and head lice. ${ }^{31}$ In vitro testing of TTO against human scabies mites demonstrated a superior result ( $60 \mathrm{~min}$ median survival time with $5 \% \mathrm{TTO}$ ) in comparison with standard treatments ( $150 \mathrm{~min}$ with ivermectin $100 \mu \mathrm{g} / \mathrm{g} ; 120 \mathrm{~min}$ with permethrin $5 \%) .{ }^{432}$ TTO has also been used as a regular adjunct treatment (Royal Darwin Hospital, treatment protocol) in combination with benzyl benzoate and oral ivermectin for the management of crusted scabies. ${ }^{433}$ Additional information on the therapeutic potential of TTO for scabies can be found in a recent review. ${ }^{21}$ However, current data are insufficient to warrant a broad recommendation for its use in the management of scabies.

The aim of this research is to determine the efficacy and safety of a TTO gel formulation $(5 \% \mathrm{v} / \mathrm{w}$ TTO) versus the active comparator permethrin cream (Lyclear, $5 \% \mathrm{w} / \mathrm{w}$ permethrin) in treating scabies infestation and preventing associated secondary bacterial infections in Aboriginal children living in remote Australia. In this study, we will assess whether the promising in vitro findings with TTO translate into a meaningful clinical benefit when it is used under 'real-world' conditions, particularly in young Aboriginal people living in rural and remote communities, where scabies is common and causes significant morbidity and mortality. If proven effective, this treatment would prevent many Aboriginal children from needing hospitalisation due to scabies-driven complications, resulting in far-reaching outcomes among the Aboriginal communities within Australia and among afflicted children internationally.

Hence, we propose to perform an international first, investigator-initiated, independently sponsored, randomised controlled trial (RCT) of a $5 \% \mathrm{v} / \mathrm{w}$ TTO gel formulation for the treatment of scabies in Aboriginal children. The idea of evaluating the safety and clinical efficacy of TTO for the management of scabies in a RCT is novel and represents the first comprehensive investigation of TTO for the management of scabies. While Aboriginal people are intended as the primary beneficiaries of the proposed research, the pattern of scabies is analogous to that observed in resource-poor and underprivileged communities globally. 


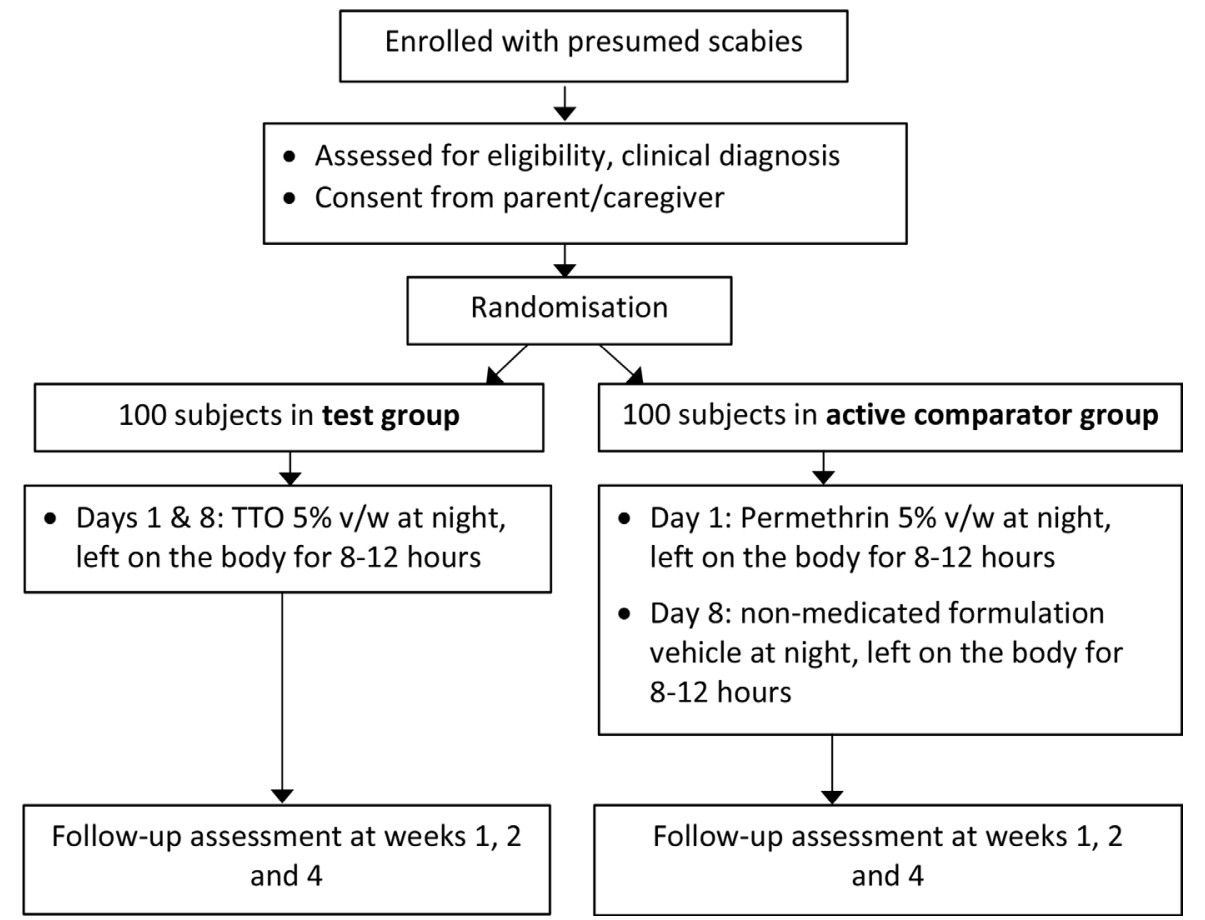

Figure 1 Study flow diagram. TTO, tea tree oil.

\section{METHODS AND ANALYSIS}

\section{Study design}

We intend to test the clinical efficacy of TTO to treat scabies infestation in children in a RCT, using a TTO-based $5 \% \mathrm{v} / \mathrm{w}$ gel similar to a TTO gel formulation previously tested in a clinical trial for cold sores. ${ }^{34}$ The $5 \%$ TTO gel contains approximately $14 \%$ poloxamer 407 gel, in addition to other excipients such as formulation stabilisers and preservatives. Permethrin 5\% cream has been selected as the active comparator due to its current status as the standard recommended therapy to treat scabies in children (and adults) in Australia. The design of this parallel group, two-arm, comparator-controlled, investigator-blind, non-inferiority trial with 1:1 allocation ratio is summarised in figure 1 . Recruitment will be staggered over 18 months (October 2017-April 2019) until 200 participants are recruited. Our reporting of the protocol conforms to the Standard Protocol Items: Recommendations for Interventional Trials (SPIRIT) 2013 checklist (online supplementary table S1 (SPIRIT Checklist) and online supplementary table S2 (WHO Trial Registration Data Set)).

The research methodology has been developed in consultation with, and is being implemented by, Aboriginal and non-Aboriginal health professionals. Extensive community consultations occurred prior to the study protocol development. Furthermore, the Aboriginal subcommittee at the study site (the Wurli-Wurlinjang Aboriginal Community Health Service) and the Aboriginal Medical Services Alliance Northern Territory reference group were involved in developing the study protocol and have endorsed the study.

\section{Study site and personnel}

The study will take place in Katherine, a remote town in Australia's Northern Territory. It will be performed in collaboration with the Wurli-Wurlinjang Health Service (WWHS), a community-controlled Aboriginal medical service. This health service began in 1972 and, based on conservative estimates, has about 6400 regular Aboriginal clients. An additional 7000 Aboriginal people who live in over 25 remote Katherine region communities are counted as occasional clients.

We plan to employ Aboriginal people as research staff to help run the study, in the course of which they will have an opportunity to acquire the skills required to manage similar work in the future. All attempts will be made to ensure that the study is explained and conducted in a culturally appropriate manner. The study documents will be translated into three locally spoken aboriginal languages: East Side/West Side Kriol, Warlpiri and Gurindji. All research staff are aware of the guidelines of Good Clinical Practice and recognise the importance of undertaking the study in a culturally appropriate way. Non-Aboriginal research staff will complete cultural awareness training and will have a strong understanding that the research should not interfere with community values.

Potentially eligible participants will be seen by a team of Wurli staff including Aboriginal health practitioners (AHPs) and general practitioners (GPs). All involved staff will follow the Central Australian Rural Practitioners Association (CARPA) code of practice from the clinic manual for primary healthcare practitioners in remote and Aboriginal health services in central and northern 
Australia. ${ }^{35}$ Study participants will be screened for eligibility by an AHP at the WWHS. Other study-related duties performed by this AHP will include participant recruitment, obtaining consent, clinical assessment, participant follow-up and site coordination, as well as coordination with other members of the study team (including interstate stakeholders). The AHP will also collaborate with two Aboriginal elders/local champions (members of the Wurli-Wurlinjang board of directors) to undertake community engagement initiatives (extensive community consultations to promote study participation). Study recruitment and treatment will be overseen by a group of senior GPs at the WWHS.

\section{Participants}

The study will be confined to patients aged between 5 and 16 years, whose legally responsible caregiver is willing for their child to participate. While there is a particularly high burden of scabies among children aged less than 5 years, the safety of TTO in this age group has not been adequately established to include them in this trial. Informed consent (online supplementary appendix A1) will be sought from caregivers during the recruitment process (prior to eligibility assessment or enrolment). Caregivers will be expected to comply with the requirements of the protocol. This includes being able and willing to be contacted by telephone after the initial assessment and being able to provide written informed consent. Furthermore, the child's assent to participate in the trial will be confirmed verbally and, if aged $\geq 12$ years, the child will be asked for written assent (online supplementary appendix A2). At the time of recruitment, the legally responsible caregiver will be asked whether the participating child will have access to regular shower facilities during the treatment course (ie, days 1 and 8), and the need for a whole-body shower before application of treatment medications will be explained.

Participant inclusion criteria are (1) the presence of typical scabietic lesions (eg, papules, nodules or vesicles) at classical sites of predilection (specifically the following 15 sites: face, head, palms, interdigits, sides of fingers, upper and lower extremities, wrists, axilla, nipple, umbilical area and/or lower abdomen, genitalia, inguinal, buttocks and back area); (2) the presence of classical burrows on clinical examination; (3) nocturnal pruritus; (4) a history of scabies among family members or similar symptoms among household contacts; and (5) access to shower facilities and a telephone.

Exclusion criteria are (1) treatment with any topical scabicidal therapy in the month before entry into the study; (2) use of any topical or systemic treatment in the week before entry; (3) confirmed or suspected immunosuppressive or immunodeficient conditions, including HIV infection; (4) receipt of more than 2 weeks of immunosuppressants or immune-modifying drugs (eg, prednisolone $>0.5 \mathrm{mg} / \mathrm{kg} /$ day) in the preceding 4 weeks; (5) the presence of scabies with an atypical presentation, such as crusted scabies; (6) the presence of any other skin disease that could alter the picture of scabies; (7) the presence of a complicated secondary bacterial infection; or (8) a known history of allergy to any of the study medications (permethrin, TTO or other essential oils).

\section{Recruitment and enrolment}

Recruitment and enrolment will occur at a single site. Participants will be recruited by an AHP while attending the WWHS clinic in Katherine. An outline of the recruitment and enrolment process and a participant-based study timeline is given in figure 2 .

Eligible participants will be offered adequate opportunities to discuss the study with the AHP on duty. The cultural integrity of the participant will be fully respected, and potential participants will not be coerced into participation. The medical care of potential participants will not be affected by their choice not to participate or to discontinue participation. Every effort will be made to ensure the potential participant fully understands the content of the Participant Information Sheet (online supplementary appendix A3) and consent forms. Information sessions in the form of short videos (video tutorials with voice-overs in Aboriginal languages, if required) will be shown to the participants and caregivers before the start of treatment to explain the nature of scabies, how it is transmitted from person to person, how to prevent recurrence and how to systematically wash their fomites (clothing, bedding or towels). The study team will also provide detailed written instructions (with the aid of flip cards) when dispensing the scabies formulations for home application by parents/caregivers and household members.

\section{Randomisation, allocation concealment}

After confirmation that the eligibility criteria have been met, the AHP will allocate participants to either the intervention or control group using a predetermined, age-stratified sequential serial number. He or she will then open the matched, sequentially numbered opaque envelope containing the treatment allocation instructions. The allocation ratio will be 1:1 active comparator (permethrin):test treatment (TTO), and the allocation sequence and treatment allocations will be concealed from trial participants and clinical assessors (AHPs) throughout the study. The simple randomisation schedule will be computer generated, and the randomisation will be kept secure (password protected) by an independent statistician (based at the University of Canberra) who is otherwise not part of the research team. The actual treatment allocation will not be disclosed to the staff members responsible for patient care, treatment, evaluation and follow-up or to the study monitors. The treatment allocation will be concealed in all participant documents.

\section{Medications and treatment}

All treatments prescribed in connection with the study will be provided free of charge to the participants. Scabies treatments for day 1 and day 8 will be provided 


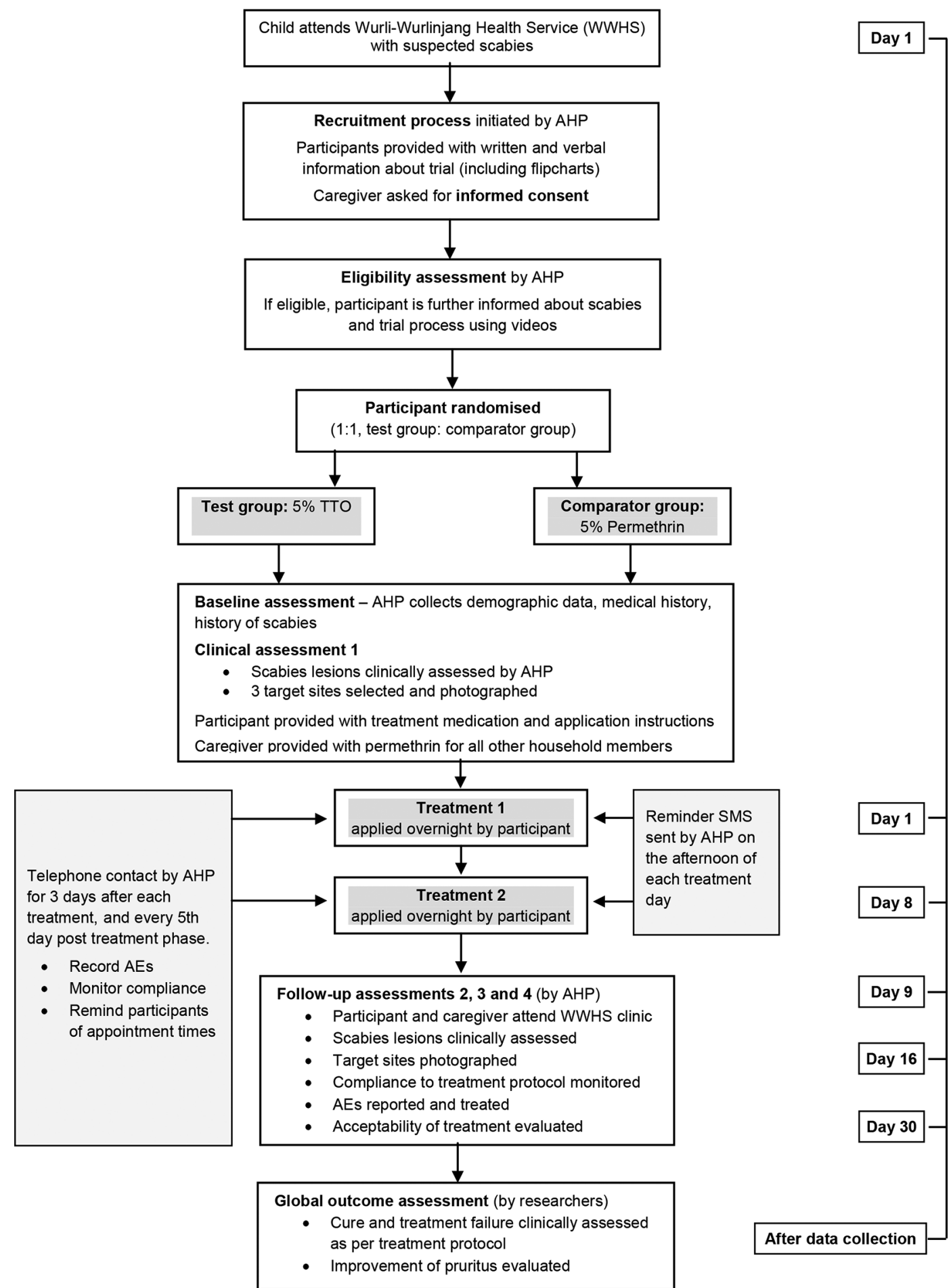

Figure 2 Overview of the trial recruitment, treatment and assessment process. AE, adverse event; AHP, Aboriginal health practitioner.

concurrently at the first visit in well-labelled containers. Treatment will consist of application of the product by the participant or caregiver wearing plastic gloves and a gown following CARPA guidelines. Participants will be instructed to shower or bathe (with non-medicated soap) and have their bed linen changed before applying the treatment. The scabies formulations will be applied to every square inch of skin, from the posterior ear folds down over the entire body. This includes the intergluteal cleft, umbilicus, skin folds, palms and soles, and webs between fingers and toes. The area behind the ears will be given special attention while avoiding eyes and the mouth. Participants and caregivers will be instructed to clip the participant's fingernails and toenails and apply the scabies formulations under nails. They will also be instructed to put on clean clothing after applying the treatment. Contaminated clothing and linens should be washed in the hot cycle of a washing machine and dried in 
the hot cycle of a dryer for 10-20 min or dried under hot sunlight. If the trial medication is washed off during hand washing, toileting or perineal care, it must be reapplied.

The scabies treatment formulations will be applied at night after an evening shower/bath. In the case of the test medication $(5 \% \mathrm{v} / \mathrm{w}$ TTO gel), the treatment will be applied on day 1 and day 8 and left on the body for 8-12hours each time. After this time, the children will shower/bathe (with non-medicated soap and water) to wash off the treatment. The comparator group medication (permethrin 5\% cream, single application only) will also be applied as directed above on day 1, followed by a placebo treatment (identical standard formulation minus permethrin) on day 8. Participants will be advised not to use or mix any other scabies treatment with trial medications.

Test formulations $(5 \% \mathrm{v} / \mathrm{w}$ TTO gel in an aqueous base) will be prepared following WHO Good Manufacturing Practices (GM, IDT Australia, VIC), and all formulations will be supplied in appropriate child-safe medicine containers, labelled with the child's identifiers and dosing (application) instructions. The trial medications will be stored in the clinic imprest at WWHS, and their supply will be supervised by the AHP. A 4-week expiry date will be set for all test formulations. On recruitment, the AHP will provide participants/caregivers (blinded) with sealed medication packs of identical appearance. The study participants and caregivers will be given additional personal protective equipment for direct patient care, including disposable long-sleeved gowns and gloves, in order to reduce the risk of transmission and prevent reinfestation. Participants will also be provided with a sealable easily identifiable biohazard bag to dispose of gloves and gowns. They will be asked to return this bag to the clinic during their follow-up visits for safe disposal. Care and precautions will be exercised while dealing with participants to minimise transmission. ${ }^{35}$

To further prevent the spread of scabies, all other family contacts will be provided therapy with a standard-approved scabicide (permethrin 5\%). This will be provided to caregivers in visually distinguishable containers with clear instructions using coloured labels to prevent medication errors. Crusted scabies-infested house contacts will be identified by questioning parents/caregivers, and the AHP will alert the Northern Territory Centre for Disease Control for the appropriate public health response.

\section{Follow-up and clinical assessment}

After giving consent, the parent or caregiver attending with the child will be asked a standard set of questions by the trained AHP to capture potentially important demographic details. Clinical evaluations (blinded to treatment allocation) will be made by experienced clinical investigators (trained AHP/Aboriginal nurse) following CARPA guidelines.

All participants will be followed up on days 9, 16 and 30 and will be thoroughly examined by the same clinical staff member (AHP) as at baseline (day 1). At each of the four visits, examination of the entire body surface will be performed. The sites of lesions will be recorded on body diagram sheets, and lesions will be photographed using a standardised protocol. ${ }^{36}$ The notations of their appearance and whether they are new lesions or residuals of original lesions will be determined by comparison with the pretreatment digital photographs. The severity of infestation will be graded based on the number of body sites showing typical scabietic lesions and recorded as mild infestation $(<6$ sites affected), moderate infestation (6-10 sites) or severe infestation ( $>10$ sites) ${ }^{37}$ The extent of lesions on the entire body will be recorded as mild ( $\leq 10$ lesions), moderate (11-49 lesions) or severe ( $\geq 50$ lesions). ${ }^{38} 39$ Trial data collection forms can be obtained on request from the corresponding author.

The primary outcome measure in this study is the clinical cure of scabies at the end of the 30-day observation period. Outcome assessments will be conducted by investigators blinded to treatment allocation. The principal investigator or coinvestigators will assess cure by referring to target site photographs and marked body diagrams after data collection is complete. Cure is defined as the absence of new lesions, and all old lesions healed, on day 30 (residual, dry, non-inflammatory papules will not be considered to be active). ${ }^{37}$ Treatment failure is defined as the presence of new or unhealed lesions on day 30 in a participant who was not considered to be cured on day 16.

The secondary outcomes that will be investigated in this study are the rate of scabies recurrence, relief of pruritus, pyodermal progression (development of secondary bacterial infections), adherence to the treatment regimen, the frequency/severity of adverse events (AEs) and patient acceptability of the treatment. Recurrence rate (or rate of scabies reinfestation) will be measured by determining the number of participants who are completely clear at day 16 and develop new lesions by day 30 . Pruritus will be evaluated using a $0-10$ visual analogue scale, where 0 represents 'no itch' and 10 represents 'worst imaginable itch'. All participants will be followed up for 3 weeks post-intervention to assess pruritus. Antipruritic medication, if needed, will be given after this period. The proportion of children with secondary bacterial complications will be determined by having trained AHPs identify and grade the severity of infected skin sores. Severity will be stratified by researchers into mild impetigo (one purulent or crusted sore and $<5$ sores in total) or severe impetigo (two or more purulent or crusted sores or $\geq 5$ sores in total) ${ }^{40}$ Participants' acceptability of treatments will be assessed during the three follow-up visits by asking participants and caregivers to rate the treatment in terms of effectiveness, side effects, convenience and overall satisfaction.

\section{Adherence and retention}

Innovative strategies will be employed to motivate participants' adherence to the treatment protocol and to increase retention. These include providing each trial 
participant with a tablet with preset reminder alarms and preloaded engaging video tutorials in relevant Aboriginal languages to facilitate the correct and easy application of trial medications. Furthermore, gift vouchers (prepaid telephone cards and/or grocery vouchers) will be given to participants who attend all follow-up visits to compensate for participant transport to the study centre for assessments. A limited supply of washing powder, childrens clothing and bed linen will be provided to study participants to better control the scabies infestation in the local communities and to facilitate the accomplishment of long-term treatment goals.

The AHP will review each participant by phone to record any apparent adverse effects and to monitor adherence to the treatment protocol, such as washing of fomites and permethrin use by other household members. Phone calls will be made daily for 3 days following each treatment application and every 5 th day post treatment phase (figure 2). The participant/caregiver will also receive reminder SMS alerts in the afternoon on each treatment day and the day before each follow-up assessment. In cases where telephone follow-up is not successful, the AHP will undertake healthcare home visits to enhance participants' adherence to the treatment protocol and to improve retention.

Participant compliance to the treatment protocols will be assessed objectively by weighing the tubes of medications prior to the supply and at the end of treatment. Parents/caregivers will be reminded by nursing staff to return the formulations and tablets at the second assessment visit. At the completion of the trial, the tablet computers will be collected and donated to local schools in the participating trial region for educational purposes.

\section{Monitoring of AEs}

During each follow-up visit and telephone call, the participants/caregivers will be asked about the occurrence of any solicited or unsolicited adverse reactions to the treatment. This will be done using a prespecified list of AEs including local adverse reactions (swelling, stinging/ burning, itching, induration, erythema, sore eyes or conjunctivitis) and systemic adverse reactions (fever, nausea, vomiting, headache and dizziness). Medically significant AEs will be documented from randomisation until the end of the study (day 30 ).

The relationship between each $\mathrm{AE}$ and the trial medication will be determined by a medical doctor according to the following definitions: (1) related: the AE follows a reasonable temporal sequence from trial medication administration and it cannot reasonably be attributed to any other cause; (2) not related: the AE is probably produced by the participant's clinical state or by other modes of therapy administered to the participant. All AEs that are observed by an investigator or member of the healthcare team, that are reported by the participant (in person or on the telephone) or that are recorded in the participant's medical records will be detailed in the case report forms (CRFs), whether or not attributed to the trial medication. AEs considered related to the trial medication will be followed either until resolution or until the event is considered stable. The following information will be recorded where available: description, date of onset and end date, severity, expectedness, assessment of relatedness to trial medication, other suspect drug or device, action taken and whether the participant is withdrawn due to $\mathrm{AE}$.

The investigator will use their clinical judgement to decide whether an $\mathrm{AE}$ is of sufficient severity to require the discontinuation of the participant's study medication. A participant may also voluntarily withdraw from treatment due to what he or she perceives as an intolerable AE. If either of these occurs, the participant will undergo an end of trial assessment and be given appropriate care under medical supervision until symptoms cease or the condition becomes stable.

\section{Feasibility}

The Katherine region is a uniquely suitable site at which to perform this trial because of its proximity to Aboriginal communities. The region is home to about 15000 Aboriginal residents, 6400 of whom are regular clients at WWHS. Our team has already established a research collaboration and agreement with the recruitment site (WWHS) to recruit participants $(n=200)$ within the proposed timeframe (18 months).

\section{Sample size}

The study sample size was calculated based on previous observational studies, in vitro data on TTO and the investigators' findings from similar trials. ${ }^{38}$ Calculations are based on the assumptions that permethrin $5 \%$ cream will have a $90 \%$ cure rate, and TTO 5\% formulation will have an $80 \%$ cure rate at 15 days. Given an estimated $10 \%$ difference in cure rate in favour of the comparator permethrin arm, a sample of 69 participants per arm (138 in total) is required to be $80 \%$ sure that the upper limit of a one-sided $95 \%$ CI will exclude a difference in favour of the permethrin arm of more than $25 \%$, thus establishing non-inferiority between TTO and permethrin. A larger sample would have permitted us to detect a smaller non-inferior difference (eg, 15\% and 10\%). However, it may not be practical to recruit a larger cohort without presenting preliminary clinical safety efficacy data, especially in children. The results will be considered significant if $\mathrm{P} \leq 0.05$. The current study will recruit 200 participants (100 in each arm, to allow for up to $30 \%$ attrition, based on our previous studies).

\section{Statistical analysis}

Data will be reported in accordance with the Consolidated Standards of Reporting Trials guidelines. A detailed analysis plan will be approved by all investigators prior to any data analysis. For each outcome, the number of evaluable children in each treatment group will be presented, and data analysts will be blinded to treatment allocation. Categorical variables will be 
summarised using frequency and percentage. Continuous variables will be first assessed for significant departures from normality using a Shapiro-Wilk test and summarised using mean and SD or median and IQR as appropriate. Significantly skewed variables will be further assessed for transformation and analysed parametrically or non-parametrically, as appropriate. Differences in cure rate between treatment arms groups will be presented with a corresponding 95\% CI and one-sided $\mathrm{P}$ value. Kaplan-Meier estimates and a log-rank test will be used to compare time-to-event outcomes by treatment arm. Cox proportional hazards regression will be used to adjust the association between treatment arm and time-to-event outcomes for known or suspected baseline confounders. The baseline comparability of groups will be further assessed, using the centre as an adjustment factor. Hazard proportionality will be assessed through analysis of scaled Schoenfeld residuals.

To reduce bias, all analyses will be based on an 'intention to treat' approach; analyses will include all randomised participants, whether treated or not, as well as any participants who have withdrawn prematurely or are poorly compliant. An a priori subgroup analysis will be performed by age group ( $<12$ years vs $12-16$ years). A test for interaction will be performed to evaluate differential treatment effects by subgroup. Sensitivity analyses will be conducted to compare: (1) the available data analysis with alternative assumptions about any missing data and (2) the 'treatment allocated' approach with 'treatment received' approach.

\section{Study management}

This study is being supported by funding provided through the University of Canberra Collaborative Indigenous Research Initiative (UC-CIRI@canberra.edu.au). The proposed Clinical Trial Management (CTM) group will consist of the coinvestigators (including the trial statistician) and the data manager. They will hold budgeted bimonthly teleconferences to review the overall conduct of the study. The trial operations team will include the site coordinator and data manager. They will teleconference monthly to discuss operational issues including data collection, cleaning and recruitment. Data will be collected on the study-specific CRFs. All data will be entered onto the password-protected online database provided by WebSpirit, stored at the University of Canberra, Discipline of Pharmacy. All coinvestigators will have access to the final trial dataset. Data queries will be raised and data cleaned by the data manager/trial statistician and site coordinators via WebSpirit. The independent Data and Safety Monitoring Committee (DSMC) will be chaired by an independent paediatric infectious disease clinician and also include a pharmacist, a statistician and a representative from the Child Health Indigenous Reference Group (WWHS). This committee will be responsible for monitoring the safety of the trial and will meet approximately every 4 months.

\section{Premature termination of the study}

This study can be terminated at any stage at the discretion of the trial sponsor (University of Canberra) or the principal investigator (JT) in consultation with the DSMC and CTM group. If the study is prematurely terminated, the principal investigator will immediately inform current trial participants and notify the relevant ethics committee within 15 days.

An interim analysis will be conducted by an independent statistician after the 100th participant has reached study day 30 to allow the DSMC to assess the ongoing appropriateness of the trial. The statistician will present the data to the DSMC in an interim report, including a descriptive analysis of AEs considered related to study treatment, the number of deaths in each study group (along with causality) and the number of participants experiencing each medically significant $\mathrm{AE}$ occurring in the first 30 days of the study. The interim analysis will be descriptive, and no hypothesis testing or presentation of $\mathrm{P}$ values for group comparisons will be made. The results of the interim analysis will remain confidential and will not be shared with study investigators unless the DSMC feels it is necessary to do so.

\section{Ethical considerations}

While preclinical studies, including hospital and laboratory-based investigations, provide grounds to expect a positive outcome for a TTO intervention, sufficient equilibrium exists to warrant an RCT of its use for scabies management in a community setting. Aboriginal liaison workers will be sought to assist in participant recruitment. Parental consent will be requested on the basis of a full explanation of known (or potential) risks and uncertainty of benefit. Participants will also be given the option to provide additional consent for their data to be used in future ancillary studies.

The privacy of participants will be protected by appropriate collection and storage of data. Participants will be identified only by initials and a participant ID number on the CRFs and in any electronic databases. The data collection forms will be stored in locked filing cabinets in a locked office at the participating clinic until the end of the study period. All documents will only be accessible by trial staff and authorised personnel. Documents containing personal information will not be stored electronically and will be anonymised as soon as it is practical to do so. Data collection forms will be held for at least 15 years on University of Canberra servers, based on National Health and Medical Research Council guidelines. These will then be destroyed.

Participants enrolled in the study will be covered by the research sponsor (University of Canberra) for indemnity and/or compensation for negligent harm or non-negligent harm arising specifically from an accidental injury and occurring as a consequence of the research subject's participation in the trial.

A protocol amendment history will be maintained and updated as necessary throughout the trial (online 
supplementary table S3). Independent audits of trial conduct will be conducted annually by authorised representatives from the sponsoring and/or host institutions. The processes audited will include participant recruitment, enrolment, allocation and assessment; reporting of harms; completeness, accuracy and timeliness of data collection; and adherence to the International Conference on Harmonisation Good Clinical Practice guidelines.

\section{Dissemination}

Research feedback at the end of the project will be in a form that is useful and understandable and include both a verbal and written summary detailing the input received and project outcomes. A suitably qualified Aboriginal person will be employed to liaise between Aboriginal communities and the research team to disseminate the study results and feedback to the local communities at large. The results will also be provided to the council and relevant community organisations. The findings of this trial will be disseminated through peer-reviewed journals and national and international scientific meetings, as well as to the participating communities. The Aboriginal reference group and the study CTM group will monitor public dissemination of any research findings. All reports will be submitted to these two groups prior to any results being published or disseminated into the public domain.

The principal investigator will coordinate dissemination of data from this study. All publications based on this study will be provided for review by each study investigator prior to submission. Authorship will be determined in line with the Uniform Requirements for Manuscripts Submitted to Biomedical Journals published by the International Committee of Medical Journal Editors. The final decision on authorship of any publication will be the responsibility of the principal investigator.

\section{Author affiliations}

${ }^{1}$ Faculty of Health, University of Canberra, Canberra, Australia

${ }^{2}$ Faculty of Health, University of Tasmania, Hobart, Australia

${ }^{3}$ School of Medicine and Pharmacology, The University of Western Australia, Perth, Australia

${ }^{4}$ Translational Renal Research Group, Harry Perkins Institute of Medical Research, Perth, Australia

${ }^{5}$ Faculty of Science, Health, Education and Engineering, University of the Sunshine Coast, Maroochydore DC, Australia

${ }^{6}$ Burnet Institute for Medical Research and Public Health, Melbourne, Australia ${ }^{7}$ University of Canberra, Canberra, Australia

${ }^{8}$ Flinders University Rural Clinic School, Flinders University, Adelaide, Australia ${ }^{9}$ Department of Child and Adolescent Health, Mackay Hospital and Health Service, West Mackay, Australia

${ }^{10}$ School of Nursing, Midwifery and Indigenous Health, Charles Sturt University, Bathurst, Australia

${ }^{11}$ Wurli-Wurlinjang Health Service, Katherine, Australia

${ }^{12}$ The Canberra Hospital, Canberra, Australia

Contributors JT conceived the study. JT, GMP and RD led the design of the quantitative common study protocol with the involvement of all authors. JT, SFW, CC, PD, TC, PC, FM, TS, KEB, AB, JM, MN and PF led the design of site-specific components. GMP and TS designed the statistical analysis plan for the protocol. TC,
JKC, PD, PC, PF, KEB and JT designed the implementation aspects of this protocol. All authors, including SK, participated in the preparation of the manuscript by providing comments on drafts written by JT and approving the final version.

Funding This work was supported by a grant received from University of Canberra Collaborative Indigenous Research Initiative (2016-2018).

Competing interests None declared.

Patient consent Caregiver consent obtained.

Ethics approval The study protocol, culturally appropriate information and consent materials were reviewed and approved by the Indigenous staff at University of Canberra (The Ngunnawal Centre), the University of Canberra Human Research Ethics Committee (HREC 16-133), WWHS Indigenous subcommittee, study reference group and the reference group from AMSANT.

Provenance and peer review Not commissioned; externally peer reviewed.

Data sharing statement The deidentified data will be made available in an openaccess repository after publishing the study results.

Open Access This is an Open Access article distributed in accordance with the Creative Commons Attribution Non Commercial (CC BY-NC 4.0) license, which permits others to distribute, remix, adapt, build upon this work non-commercially, and license their derivative works on different terms, provided the original work is properly cited and the use is non-commercial. See: http://creativecommons.org/ licenses/by-nc/4.0/

(c) Article author(s) (or their employer(s) unless otherwise stated in the text of the article) 2018. All rights reserved. No commercial use is permitted unless otherwise expressly granted.

\section{REFERENCES}

1. Currie BJ, Harumal P, McKinnon M, et al. First documentation of in vivo and in vitro ivermectin resistance in Sarcoptes scabiei. Clin Infect Dis 2004;39:e8-12.

2. Mika A, Reynolds SL, Pickering D, et al. Complement inhibitors from scabies mites promote streptococcal growth--a novel mechanism in infected epidermis? PLoS Negl Trop Dis 2012;6:e1563.

3. Thomas J, Peterson GM, Walton SF, et al. Scabies: an ancient global disease with a need for new therapies. BMC Infect Dis 2015;15:250.

4. Walton SF, McKinnon M, Pizzutto S, et al. Acaricidal activity of Melaleuca alternifolia (tea tree) oil: in vitro sensitivity of sarcoptes scabiei var hominis to terpinen-4-ol. Arch Dermatol 2004;140:563-6.

5. Bouvresse S, Chosidow O. Scabies in healthcare settings. Curr Opin Infect Dis 2010;23:111-8.

6. Hay RJ, Estrada Castanon R, Alarcon Hernandez H, et al. Wastage of family income on skin disease in Mexico. BMJ 1994;309:848.

7. Anon. One Disease At A Time (AU). Sydney, NSW, 2017. http:// onedisease.org/why-scabies/. (accessed 30 May 2017).

8. Hay RJ. Scabies and pyodermas---diagnosis and treatment. Dermatol Ther 2009;22:466-74.

9. Burkhart CG, Burkhart CN, Burkhart KM. An epidemiologic and therapeutic reassessment of scabies. Cutis 2000;65:233-40.

10. Chosidow O. Clinical practices. Scabies. N Engl J Med 2006;354:1718-27.

11. Currie BJ, McCarthy JS. Permethrin and ivermectin for scabies. $N$ Engl J Med 2010;362:717-25.

12. Hay RJ, Steer AC, Engelman D, et al. Scabies in the developing world--its prevalence, complications, and management. Clin Microbiol Infect 2012;18:313-23.

13. Kemp DJ, Walton SF, Harumal P, et al. The scourge of scabies. Biologist 2002;49:19-24.

14. Mounsey KE, Holt DC, McCarthy J, et al. Scabies: molecular perspectives and therapeutic implications in the face of emerging drug resistance. Future Microbiol 2008;3:57-66.

15. Mounsey KE, Holt DC, McCarthy JS, et al. Longitudinal evidence of increasing in vitro tolerance of scabies mites to ivermectin in scabies-endemic communities. Arch Dermatol 2009;145:840-1.

16. Pasay C, Walton S, Fischer K, et al. PCR-based assay to survey for knockdown resistance to pyrethroid acaricides in human scabies mites (Sarcoptes scabiei var hominis). Am J Trop Med Hyg 2006;74:649-57.

17. Terada $\mathrm{Y}$, Murayama N, Ikemura $\mathrm{H}$, et al. Sarcoptes scabiei var. canis refractory to ivermectin treatment in two dogs. Vet Dermatol 2010;21:608-12.

18. de Beer G, Miller MA, Tremblay L, et al. An outbreak of scabies in a long-term care facility: the role of misdiagnosis and the 
costs associated with control. Infect Control Hosp Epidemiol 2006;27:517-8.

19. Owusu-Edusei K, Chesson HW, Gift TL. The economic burden of pediculosis pubis and scabies infections treated on an outpatient basis in the United States: evidence from private insurance claims data, 2001-2005. Sex Transm Dis 2009;36:297-9.

20. Carson CF, Hammer KA, Riley TV. Melaleuca alternifolia (Tea Tree) oil: a review of antimicrobial and other medicinal properties. Clin Microbiol Rev 2006;19:50-62.

21. Thomas J, Carson CF, Peterson GM, et al. Therapeutic potential of tea tree oil for scabies. Am J Trop Med Hyg 2016;94:258-66.

22. Greig JE, Carson CF, Stuckey MS, et al. Skin sensitivity testing for tea tree oil: RIRDC project no. UWA-42A. Australia 1999.

23. Markum E, Baillie J. Combination of essential oil of Melaleuca alternifolia and iodine in the treatment of molluscum contagiosum in children. J Drugs Dermatol 2012;11:349-54.

24. Aspres N, Freeman S. Predictive Testing for Irritancy and Allergenicity of Tea Tree Oil in Normal Human Subjects. Exogenous Dermatology 2003;2:258-61.

25. Hammer KA, Carson CF, Riley TV, et al. A review of the toxicity of Melaleuca alternifolia (tea tree) oil. Food Chem Toxicol 2006;44:616-25.

26. Williamson EM, Priestley CM, Burgess IF. An investigation and comparison of the bioactivity of selected essential oils on human lice and house dust mites. Fitoterapia 2007;78:521-5.

27. Gao YY, Di Pascuale MA, Elizondo A, et al. Clinical treatment of ocular demodecosis by lid scrub with tea tree oil. Cornea 2007:26:136-43.

28. Gao YY, Di Pascuale MA, Li W, et al. In vitro and in vivo killing of ocular Demodex by tea tree oil. Br J Ophthalmol 2005;89:1468-73.

29. Mägi E, Järvis T, Miller I. Effects of different plant products against pig mange mites. Acta Veterinaria Brno 2006;75:283-7.
30. Sherry E, Sivananthan S, Warnke PH, et al. Topical phytochemicals used to salvage the gangrenous lower limbs of type 1 diabetic patients. Diabetes Res Clin Pract 2003;62:65-6.

31. Di Campli E, Di Bartolomeo S, Delli Pizzi P, et al. Activity of tea tree oil and nerolidol alone or in combination against Pediculus capitis (head lice) and its eggs. Parasitol Res 2012;111:1985-92.

32. Walton SF, Myerscough MR, Currie BJ. Studies in vitro on the relative efficacy of current acaricides for Sarcoptes scabiei var. hominis. Trans R Soc Trop Med Hyg 2000;94:92-6.

33. Davis JS, McGloughlin S, Tong SY, et al. A novel clinical grading scale to guide the management of crusted scabies. PLoS Negl Trop Dis 2013;7:e2387.

34. Carson CF, Ashton L, Dry L, et al. Melaleuca alternifolia (tea tree) oil gel $(6 \%)$ for the treatment of recurrent herpes labialis. J Antimicrob Chemother 2001:48:450-1.

35. CARPA. CARPA standard treatment manual. Alice Springs: Central Australian Rural Practitioners Association, 1994.

36. Bowen AC, Burns K, Tong SY, et al. Standardising and assessing digital images for use in clinical trials: a practical, reproducible method that blinds the assessor to treatment allocation. PLoS One 2014:9:e110395

37. Castillo AL, Osi MO, Ramos JD, et al. Efficacy and safety of Tinospora cordifolia lotion in Sarcoptes scabiei var hominis-infected pediatric patients: A single blind, randomized controlled trial. $J$ Pharmacol Pharmacother 2013;4:39-46.

38. Chouela EN, Abeldaño AM, Pellerano G, et al. Equivalent therapeutic efficacy and safety of ivermectin and lindane in the treatment of human scabies. Arch Dermatol 1999;135:651-5.

39. Elmogy $\mathrm{M}$, Fayed $\mathrm{H}$, Marzok $\mathrm{H}$, et al. Oral ivermectin in the treatment of scabies. Int J Dermatol 1999;38:926-8.

40. Bowen AC, Tong SYC, Andrews RM, et al. Short-course oral cotrimoxazole versus intramuscular benzathine benzylpenicillin for impetigo in a highly endemic region: an open-label, randomised, controlled, non-inferiority trial. The Lancet 2014;384:2132-40. 